\title{
Do Antepartum Herpes Simplex Virus Cultures Predict Intrapartum Shedding for Pregnant Women With Recurrent Disease?
}

\author{
S.M. Garland, ${ }^{1 *}$ T.N. Lee, ${ }^{2}$ and S. Sacks ${ }^{2}$ \\ ${ }^{1}$ Department of Microbiology, Royal Women's Hospital, Melbourne, Australia \\ ${ }^{2}$ Department of Pharmacology \& Therapeutics, Faculty of Medicine, University of British Columbia \\ \& Viridae Clinical Sciences Inc., Vancouver, BC, Canada
}

\begin{abstract}
Objective: To examine antenatal screening as a predictor of intrapartum shedding of herpes simplex virus (HSV) and to determine its usefulness in guiding the appropriate route of delivery for patients with recurrent HSV in pregnancy.

Methods: A population of 198 pregnant women with a history of recurrent genital HSV were cultured in the last weeks of their pregnancy by specially-trained personnel and intrapartum by their delivering attendants.

Results: Of cultures from a total of 906 antenatal visits, $17 \%$ were culture positive, with an asymptomatic shedding rate of $3.4 \%$. Asymptomatic shedding occurred in $12.6 \%$ of women. Over the 8-week antepartum period, viral culture-positivity rates for each visit ranged from $11 \%$ to $19.5 \%$. This provided an expected delivery culture-positivity rate of $15.3 \%$. However, actual intrapartum viral culture positivity occurred in only three of 191 women $(1.5 \% ; P<0.001)$. Because previous studies have suggested antepartum culture positivity fails to predict intrapartum viral shedding, evaluations, including cultures, as well as predictive values for subsequent culture positivities, were determined under the supervision of an infectious disease specialist. Under these conditions, positive predictive values were $59 \%$ when the interval between visits was 2 days, but only $19 \%$ when days between visits were $>2(P<0.0001)$. No cases of neonatal herpes were seen in this population, although cesarean deliveries were performed in $31 \%$ of the patient population, with genital herpes as the indication for $56 \%$ of those.

Conclusions: Antepartum serial screening by viral culture is not predictive of an infant's risk of intrapartum viral exposure when conducted at weekly intervals. However, more frequent assessments of patients can be predictive of an infant's exposure risk to HSV; for patients with frequent recurrent disease near term or primary infection in pregnancy, frequent late antepartum screening may be appropriate. Infect. Dis. Obstet. Gynecol. 7:230-236, 1999. @ 1999 Wiley-Liss, Inc.
\end{abstract}

KEY WORDS

herpes simplex virus; prevention; pregnancy

$\mathrm{N}$ eonatal herpes simplex virus (HSV) infection is largely acquired intrapartum and, even with prompt recognition and treatment, is associated with significant morbidity and mortality. ${ }^{1-3}$ Maternal infection is unrecognized in the majority of cases. ${ }^{4,5}$ In an attempt to reduce the incidence of this disease, the American Academy of Pediatrics in 1980 recommended serial viral screening in the last weeks of pregnancy for all women with a past history of genital herpes. ${ }^{6}$ Avoidance of vaginal de-

*Correspondence to: Associate Professor S.M. Garland, Microbiology Department, Royal Women's Hospital, Grattan Street, Carlton, Victoria, Australia 3053. E-mail: garlands@cryptic.rch.unimelb.edu.au 
livery was recommended to prevent transmission of virus to the infant in the setting of either clinical or virologic evidence of active infection. Arvin et al., however, demonstrated that weekly antepartum cultures in the third trimester were not actually predictive of asymptomatic intrapartum shedding. ${ }^{7}$ This has led to revision of the guidelines, with the elimination of serial antepartum viral screening. 8 Further arguments against serial screening of symptomatic patients include the premise that most women with genital HSV are infected subclinically. Furthermore, Brown et al. have demonstrated that neonatal risk is highest in the setting of recent acquisition of infection. ${ }^{9}$ In fact, Randolph et al. have recently suggested the elimination of cesarean delivery for active recurrent disease intrapartum. ${ }^{10}$ Nevertheless, recommendations of these authors have not been widely accepted as yet, ${ }^{11}$ and recurrent disease still accounts for up to $50 \%$ of neonatal cases. Hence, the current management of patients with recurrent genital herpes remains unsettled, the decision regarding cesarean delivery being largely left to clinical judgment regarding evident symptomatic lesions in labour. As a compromise, some investigators have chosen to suppress recurrences with oral acyclovir during the final 2-4 weeks of gestation, ${ }^{12,13}$ hoping to avoid cesarean deliveries.

During the mid-1980s, a group of patients considered at high risk for genital herpes were referred to a university teaching hospital clinic for prenatal assessment and follow up. Because of the recent changes in our understanding of the pathogenesis of neonatal infection, we analysed data collected from that period to extend our understanding of the natural history of the disease. We report here our experience of antenatal screening to predict intrapartum shedding and its value in guiding the appropriate route of delivery for patients with recurrent herpes in pregnancy.

\section{METHODS \\ Patient Population}

The study population consisted of 198 pregnant women referred from May 1985 to July 1987 to the University of British Columbia Herpes Clinic, Vancouver, Canada, by their local medical practitioners because the women had a previous history of genital HSV, previous genital culture positive for HSV, or their partners had a history of HSV. After written consent was obtained, patients completed a questionnaire detailing patient demography, pregnancy and sexual history, and past sexually-transmitteddisease history with detailed questioning for oral and genital herpes (including a photographic questionnaire). Serum was obtained from each woman at initial assessment for HSV type-specific antibodies.

Clinical evaluation of patients occurred at weeks 32,34 , and 36 of gestation and weekly to delivery according to standard practice at that time. Evaluations were performed by an infectious disease specialist, and at each visit symptoms and signs of any recent genital HSV were documented. In addition, cultures were taken from any apparent clinical le$\operatorname{sion}(\mathrm{s})$, or, if no lesions were present, a general sweep from the external genital area (a Dacrontipped metal swab previously moistened in normal saline was wiped firmly over the pubic area, clitoris, periurethral area, labia minora and majora, fourchette, perineal and perianal areas), and a separate swab was also collected from the ectocervix.

At the conclusion of each visit, a recommendation was made to the attending physician as to the ideal mode of delivery. Where no clinical disease was evident, vaginal delivery was recommended, whereas for an active episode (symptomatic/ asymptomatic), cesarean delivery was considered the ideal delivery mode, if labour intervened. For those with an active episode antepartum, more frequent follow up of the patient was arranged at the Herpes Clinic, until a return to a culture-negative and asymptomatic clinical status was achieved. At birth, repeat cultures (as described above) were performed of the upper and lower genital tract by the attending obstetrician or physician. In addition, the fresh placenta, the infant (mouth, axilla, groin), and, in the case of those delivered by cesarean delivery, amniotic fluid, were also cultured for HSV. A research nurse collected individual patient details in labour and baby outcome at birth, as well as follow up at 6 weeks postpartum, with a telephone call to parents to inquire regarding the baby's health.

\section{Viral Culture}

After sampling, swabs were placed into viral transport medium which consisted of Medium 199 with Hank's balanced salt solution containing $1 \%$ inactivated fetal calf serum plus antibiotics (penicillin, 
streptomycin, and amphotericin B). Specimens were transported daily for inoculation in duplicate onto human foreskin fibroblast monolayers and processed by standard methods. ${ }^{14}$ Monolayers were examined daily for development of cytopathic effects, when they were stained with HSV type-specific monoclonal antibodies (Syva, Palo Alto, CA) for confirmation and typing as previously described. ${ }^{15}$

\section{Viral Serology}

Antibodies to HSV (type specific) were detected by microneutralization and Western blot assay. ${ }^{16}$

\section{RESULTS}

Demographic details of the patient population are outlined in Table 1. For the 8 -week antepartum period during which the 198 patients were regularly assessed clinically and by culture, 906 total visits were made. For the total visits, 884 had completed data for both symptoms and signs, whereas for symptoms or for signs, 898 and 903 , respectively (Table 2). The median and mean number of visits were 4.0 (interquartile, 3-6) and 4.6 (standard deviation [SD], 2.6), respectively; the median interval between visits was 7.0 days (interquartile, 4-9), mean, 8.2 (SD, 9.9). Between the last antenatal visit to delivery, the median number of days was 4.0 (interquartile, 2-8), mean, 8.9 (SD, 27.8). Overall $88.7 \%$ of patients were positive by Western blot for HSV type-2 antibodies (with or without HSV type-1 antibodies); $62 \%$ were positive for HSV type- 2 antibodies alone, and $8.7 \%$ were positive for HSV type-1 alone. ${ }^{16}$

Of the 198 patients, 191 had at least one followup visit. Details of clinical lesions and of culture results for all the antepartum visits and of the ultimate visit prior to delivery are depicted in Table 2. It is evident that symptoms were reported in $20 \%$ and signs consistent with HSV evident in $28 \%$ of all visits, whilst $17 \%$ of all visits were positive by culture. Overall, 94 patients (48\%) had HSV confirmed by culture (for 154 visits), and $97 \%$ of these isolates were HSV type 2. Patients were either symptomatic (as defined by prodrome, redness, blister, ulcer, or crust) or had signs consistent with HSV (macule, papule, vesicle, pustule, or ulcer) in $89 \%$ of these visits. Twenty-four women $(12.6 \%)$ shed HSV asymptomatically. Six of these women shed on two occasions. Therefore, the asymptomatic shedding rate for the total antepartum visits
TABLE I. Demographic characteristics of the pregnant population

\begin{tabular}{|c|c|c|}
\hline Variable & $\mathrm{n}( \pm \mathrm{SD})$ & $\%$ \\
\hline Age at Ist pregnancy & $26.3 \pm 5.3$ & \\
\hline Age at Ist sexual contact & $18.1 \pm 2.7$ & \\
\hline Number of lifetime sexual partners & $14.6 \pm 8.5$ & \\
\hline Age at enrollment (years) & $31.1 \pm 4.3$ & \\
\hline \multicolumn{3}{|l|}{ Number of previous pregnancies } \\
\hline 0 & 82 & 41.4 \\
\hline $1-3$ & 107 & 54.5 \\
\hline$>3$ & 7 & 3.6 \\
\hline \multicolumn{3}{|l|}{ Marital status } \\
\hline Never married & 13 & 6.9 \\
\hline Married/common-law & $|7|$ & 90.5 \\
\hline Divorced/separated & 4 & 2.1 \\
\hline Widowed & 1 & 0.5 \\
\hline \multicolumn{3}{|l|}{ Racial background } \\
\hline Caucasian & 182 & 95.3 \\
\hline Black & 0 & 0 \\
\hline Other & 9 & 4.7 \\
\hline \multicolumn{3}{|l|}{ Income (thousand dollars) } \\
\hline$<25$ & 43 & 23.0 \\
\hline $25-49.9$ & 88 & 47.1 \\
\hline$>50$ & 56 & 29.9 \\
\hline \multicolumn{3}{|l|}{ Education } \\
\hline High school or less & 25 & 13.2 \\
\hline Some technical school & 24 & 12.7 \\
\hline Some university & 140 & 74.1 \\
\hline \multicolumn{3}{|l|}{ Personal history of genital herpes } \\
\hline Yes & 168 & 88.4 \\
\hline No & 4 & 2.1 \\
\hline Maybe & 18 & 9.5 \\
\hline \multicolumn{3}{|l|}{ History of oral cold sores } \\
\hline Yes & 67 & 35.3 \\
\hline No & 109 & 57.3 \\
\hline Maybe & 14 & 7.4 \\
\hline \multicolumn{3}{|l|}{ Sex with known HSV-infected person } \\
\hline No & 65 & 36.3 \\
\hline 1-2 times & 48 & 26.8 \\
\hline Often & 66 & 36.9 \\
\hline \multicolumn{3}{|l|}{ Previous Sexually Transmitted Disease } \\
\hline Yes & 54 & 27.8 \\
\hline No & 142 & 72.2 \\
\hline
\end{tabular}

was $3.4 \%$ (30/884). Of these 30 asymptomatic shedding episodes, $86.7 \%$ (26) were from the external anogenital area, and $3.4 \%$ (1) were from the cervix, while the remainder were from the usual lesion site. The patient who shed from the cervix also shed from the external anogenital area of the labia. Twenty-five percent (6/24) of those women with asymptomatic shedding had no signs or symptoms of genital herpes for at least 4 weeks (mean, 27 days; range, 7-72) prior to the positive culture. Similarly, most subjects with asymptomatic shedding $(66.4 \%$ [16/24]) did not have subsequent symptoms or signs of genital HSV. Of the $33.3 \%$ $(8 / 24)$ who did subsequently have symptoms or 
TABLE 2. Description of findings at antenatal visits

\begin{tabular}{|c|c|c|c|c|}
\hline \multirow[b]{2}{*}{ Variable } & \multicolumn{2}{|c|}{$\begin{array}{c}\text { Total } \\
\text { antenatal visits } \\
\end{array}$} & \multicolumn{2}{|c|}{$\begin{array}{c}\text { Final } \\
\text { antenatal visit }\end{array}$} \\
\hline & $\mathrm{n}$ & $\%$ & $\mathrm{n}$ & $\%$ \\
\hline Total prenatal visits & 906 & & $\left.19\right|^{\mathrm{a}}$ & \\
\hline Symptoms of herpes & $182 / 898$ & 20.3 & $26 / 191$ & 13.6 \\
\hline Signs of herpes & $251 / 903$ & 27.8 & $30 / 190$ & 15.8 \\
\hline \multicolumn{5}{|l|}{ Lesion site frequency } \\
\hline Cervix & 1 & 0.1 & 0 & 0 \\
\hline Labia majora & 88 & 10.7 & 11 & 6.4 \\
\hline Labia minora & 32 & 3.9 & 2 & 1.2 \\
\hline Perineal & 28 & 3.4 & 4 & 2.3 \\
\hline Perianal & 30 & 3.7 & 4 & 2.3 \\
\hline Buttocks & 33 & 4.0 & 5 & 2.9 \\
\hline Other genital & 43 & 5.2 & 4 & 2.3 \\
\hline Remote sites & 13 & 1.6 & 3 & 1.7 \\
\hline \multicolumn{5}{|l|}{ Lesion stage } \\
\hline Macule & II & 4.2 & 4 & 12.1 \\
\hline Papule & 20 & 7.7 & 1 & 3.0 \\
\hline Vesicle & 18 & 6.9 & 2 & 6.1 \\
\hline Pustule & 22 & 8.5 & 2 & 6.1 \\
\hline Ulcer & 119 & 45.8 & 13 & 39.4 \\
\hline Crust & 33 & 12.7 & 4 & 12.1 \\
\hline Healed & 37 & 14.2 & 7 & 21.2 \\
\hline Positive culture, any sites & $154 / 906$ & 17.0 & $16 / 19 \mid$ & 8.4 \\
\hline Genital culture positive & $121 / 892$ & 13.4 & $10 / 188$ & 5.2 \\
\hline Cervix culture positive & $3 / 756$ & 0.3 & $1 / I 74$ & 0.5 \\
\hline Usual skin site positive & $25 / 83$ & 2.9 & $5 / 15$ & 2.7 \\
\hline Asymptomatic shedding rate & $30 / 884$ & 3.4 & - & - \\
\hline
\end{tabular}

aDelivery details only available for 191 patients.

signs (mean, 12.6 days; range, $2-44)$, six (75\%) were culture positive.

As depicted in Figure 1, the ability of an antepartum culture positive for HSV to predict a subsequent positive result (i.e., positive predictive value) was $59 \%(30 / 51)$ when the interval between visits was $\leq 2$ days, but only $19 \%(53 / 257)$ when days between visits were $>2(P<0.0001)$. Clinical evidence of HSV was less predictive of a subsequent positive culture result. When the interval between visits was $\leq 2$ days, clinical prediction of a positive culture (i.e., positive predictive value) was $40 \%(27 / 67)$, whereas it was $17 \%(59 / 355)$ for $>2$ days $(P<0.0001)$.

At the final antenatal visit before labour, symptoms were reported in $13.6 \%$, whilst signs of HSV were evident in $15.8 \%$, and cultures were positive in $8.4 \%$ (Table 2). Intrapartum, a similar number of women reported symptoms (11.5\%), and signs were evident on clinical examination in $9.5 \%$. However, cultures taken by the patients' attending physician intrapartum were positive in only $1.5 \%(3 / 191)$ of cases (Table 3). All three had previous signs and symptoms of HSV and were Western-blot-positive for HSV type 2 antibodies, and two were culture positive antepartum. In two of these cases, patients reported symptoms of herpes in labour, and active herpetic lesions were evident on exam. Both cases were delivered by cesarean delivery with a favourable outcome. The third case was asymptomatic at term and was delivered vaginally without adverse outcome. Of the 24 women who had asymptomatic excretion of HSV during pregnancy, three had signs or symptoms intrapartum, and none were culture positive.

The results of HSV culture positivity for the final six antepartum visits (as collected by the staff of the Herpes Clinic) together with the actual rate at delivery (as collected by the patients' own care provider) are shown in Table 3. As the intrapartum culture rate $(1.5 \%)$ is at variance with the foregoing antepartum weekly rates (11.4-19.5\%), an estimated intrapartum positivity was calculated based on these antepartum results. Two methods of estimation were performed. A crude estimate was made by taking an average percentage of the culture-positive rate of the previous six visits and was found to be $16.1 \%$. The second estimate was a weighted average of the probability of culture positivity for each patient at delivery, based on the number of their previous visits. These probabilities were weighted by the number of visits for each patient, then the average was calculated. The anticipated delivery culture-positivity rate was calculated at $15.3 \%$ had the Herpes Clinic staff taken these specimens. Calculations were adjusted to eliminate any selection bias; i.e., assessment was only made for those patients initially negative by culture who became positive. Cases positive at the initial antepartum assessment were excluded. All analyses were carried out using a Statistical Analysis System.

For antepartum visits that occurred within 7 days of delivery (130), viral shedding occurred in 13 $(10 \%)$ (Table 4$)$. The asymptomatic shedding rate of these visits was $3 \%$. Of those advised to have a cesarean delivery on the basis of active lesion or positive HSV cultures, $85 \%$ (11/13) delivered by this method. Overall, cesarean deliveries were performed for $31 \%$ of patients, with genital HSV being the indication in $56 \%$. Recommended delivery plans based on the antepartum screening highly correlated with the actual delivery method and the culture results of the final antepartum assessment 


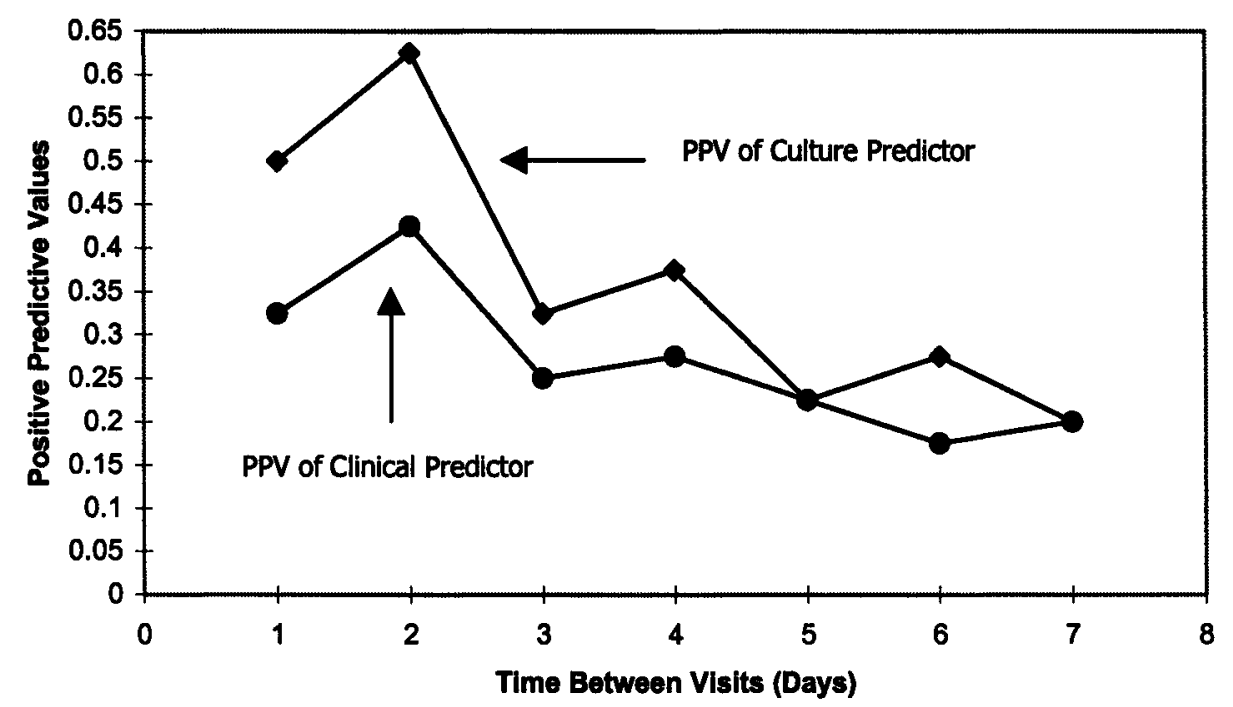

Fig. I. Predicting HSV shedding from the genital tract. Positive predictive value (PPV) ( $\square$ ) of culture as the predictor. Positive predictive value $(\Theta)$ of clinical assessment is the predictor of herpes shedding from the genital tract in subsequent patient visit.

TABLE 3. Actual and estimated culture positivity at delivery based on previous antepartum follow up

\begin{tabular}{|c|c|c|c|c|}
\hline Visit & $\begin{array}{c}\text { No. } \\
\text { positive/total }\end{array}$ & $\%$ & $\begin{array}{c}\text { No. } \\
\text { symptomatic/total } \\
\text { positive HSV } \\
\text { isolations }\end{array}$ & $\%$ \\
\hline 6 & $8 / 42$ & 19.5 & $7 / 8$ & 87.5 \\
\hline 5 & $9 / 57$ & 15.8 & $6 / 9$ & 66.8 \\
\hline 4 & $16 / 75$ & 21.3 & $11 / 16$ & 68.8 \\
\hline 3 & $|0 / 9|$ & 11.0 & $7 / 10$ & 70.0 \\
\hline 2 & $|8 / 10|$ & 17.8 & $12 / 18$ & 66.8 \\
\hline 1 & $12 / 105$ & 11.4 & $10 / 12$ & 83.3 \\
\hline Delivery & $3 / 191$ & $1.5^{*}$ & $2 / 3$ & \\
\hline \multicolumn{5}{|c|}{ Estimated at delivery } \\
\hline Crude & $31 / 191$ & $16.1 *$ & $22 / 31$ & 71.0 \\
\hline Weighted & $29 / 191$ & $15.3^{*}$ & $22 / 29$ & 79.0 \\
\hline
\end{tabular}

$* p<0.001$ (trend estimate).

$(P<0.001)$ (Table 4). There were no cases of neonatal herpes detected, and all baby and placental swabs taken at delivery were negative by viral culture.

\section{DISCUSSION}

In this patient population with histories of recurrent genital herpes, the observed culture-positivity rate for HSV for the 6-week period prior to delivery was consistently high, ranging from $11 \%$ to $19.5 \%$. An unexpected finding in this study was the low culture-positivity rate when nonspecialized personnel took genital swabs for culture. This unanticipated occurrence confounded the intrapartum cul-
TABLE 4. Comparison between findings at final antepartum ( $\leq 7$ days) visit to recommended and actual delivery plan ${ }^{\mathrm{a}}$

\begin{tabular}{|c|c|c|c|c|c|c|c|}
\hline \multirow[b]{3}{*}{ Variable } & \multicolumn{6}{|c|}{ Last plan for mode of delivery } & \multirow[b]{3}{*}{$P$ value } \\
\hline & \multicolumn{2}{|c|}{ Plan 1} & \multicolumn{2}{|c|}{ Plan 2} & \multicolumn{2}{|c|}{ Plan 3} & \\
\hline & $\mathbf{n}$ & $\%$ & $\mathbf{n}$ & $\%$ & $\mathrm{n}$ & $\%$ & \\
\hline \multicolumn{8}{|c|}{ Culture at the last visit } \\
\hline Negative & 11 & 9.4 & 12 & 10.3 & 94 & 80.3 & \\
\hline Positive & 11 & 84.6 & 0 & 0.0 & 2 & 15.4 & $<0.001$ \\
\hline \multicolumn{8}{|c|}{ Actual delivery } \\
\hline Vaginal & 4 & 4.6 & 4 & 4.6 & 79 & 90.8 & \\
\hline Cesarean & 18 & 43.9 & 8 & 19.5 & 15 & 36.6 & $<0.001$ \\
\hline
\end{tabular}

aPlan I: Cesarean until change in HSV status.

Plan 2: Cesarean until culture negative at specified week of gestation. Plan 3: Normal vaginal delivery.

ture rate, which was at variance with the preceding antepartum shedding rate of herpes for the patient population. Only three patients were culture positive intrapartum and two of these in fact had clinical lesions and were delivered by cesarean delivery accordingly. The third patient shed HSV asymptomatically and delivered vaginally; her baby was culture negative and had a good outcome.

Over the 8-week antepartum period, symptoms consistent with HSV occurred in $20 \%$ of visits, and these were culture positive in $55.7 \%$. These findings are similar to those reported by Vontver, where $56 \%$ of recurrent clinical lesions were culture positive. ${ }^{17}$ In our study group, $17 \%$ of antepartum visits were culture positive and the asymptomatic shedding rate was $3.4 \%$ (external genital, 
$96.6 \%$; cervical, $3.4 \%$ ). Overall, $12.6 \%$ of patients shed asymptomatically antepartum. This figure contrasts with that of other similar studies in which asymptomatic excretion has been reported in antepartum patients at around $4 \%$ and at a shedding rate of $0.35-0.75 \% .^{18}$ We believe this tenfold difference in shedding detection relates predominantly to expert personnel taking the clinical samples rather than others. This is further supported by the unexpectedly low intrapartum shedding rate (samples collected by delivery ward staff) found in our study, compared with that anticipated by antepartum rates (samples collected by personnel experienced in infectious disease). A further explanation for these findings may be the dilution effect on viral load by the flushing effect of amniotic fluid following rupture of the chorioamniotic membranes. The actual rate of shedding intrapartum of $1.5 \%$ for this study is similar to those previously reported (range, $0.35-2.4 \%)^{7,18,19}$

No cases of neonatal herpes were seen in this study and no babies cultured positive at birth. The overall cesarean delivery rate was $31 \%$, HSV being the indication in $56 \%$. The recommendation to deliver by cesarean delivery, based on the last antepartum visit culture, correlated well with the actual delivery mode. Of those cultured within 7 days prior to delivery, $10 \%$ were positive $(13 / 130)$, and $84.6 \%(11 / 13)$ who had been advised to have a cesarean delivery on these grounds complied.

Data from our study confirm the findings of Arvin et al. ${ }^{7}$ that early preterm weekly cultures from patients are not helpful in predicting shedding at delivery. Those data were obtained by clinicians in the field, which, in light of our findings, may not have been a sensitive marker. Further, their finding of lack of predictability is not surprising and reflects inherent problems with HSV culture, which, at best, takes 48 hours to produce a result. Also, recurrent lesions shed for a short time (3-4 days); consequently, by the time a result is obtained from a laboratory, it poorly reflects the situation at that time. However, our study did show that antepartum exams were highly predictive of subsequent outcome where visits between prediction and outcome were 2 days but not predictive when the interval between visits was more than 2 days. Therefore, we believe that appropriately and carefully collected antepartum serial cultures may still have a role in selected patients, i.e., those suf- fering frequent recurrences near term. In this scenario, viral cultures every few days to demonstrate cessation of viral shedding could obviate the need for cesarean delivery.

The neonatal attack rate for women who deliver vaginally in the presence of recurrent genital HSV lesions is very low, on the order of $<2 \%{ }^{20}$ In contrast, women with primary genital HSV near or at delivery have a neonatal transmission rate 35$80 \%{ }^{4,9,10,21}$ With such a very low rate of neonatal HSV from recurrent maternal infections, some advise covering genital lesions and allowing delivery vaginally as, the morbidity of cesarean delivery is greater. ${ }^{10,22}$ Yet, cervical shedding in association with recurrent external HSV lesions, although less frequent to primary infections $(85 \%)$ is reported to occur in $12-16 \%$ of cases. ${ }^{23}$ Moreover, as neonatal HSV carries the risk of significant morbidity and mortality, even with acyclovir treatment, we believe, as do others, ${ }^{24}$ that cesarean delivery is the appropriate form of delivery when clinical maternal genital lesions are apparent.

Asymptomatic shedding data from our study are consistent with the findings of Wald et al. in a nonpregnant population. ${ }^{25}$ They also demonstrated a much higher frequency of reactivation based on detection by polymerase chain reaction. Accordingly, wide inconsistency exists in any management strategy that requires recognition of symptoms. Some studies have adopted acyclovir suppression, now established to reduce both symptomatic and asymptomatic viral shedding rates outside of pregnancy, as an alternative strategy. Unfortunately, definitive answers regarding efficacy against both maternal shedding and neonatal infection are not yet available. Furthermore, while the safety of acyclovir in pregnancy is likely, it is unproven. In our view, there is little reason to doubt that acyclovir will predictably, but not incompletely, suppress viral shedding and symptomatic recurrent infection in pregnancy and, therefore, will likely reduce cesarean delivery rates for women with high recurrence frequency rates. This would appear to offer a strong cost benefit and would potentially obviate surgical morbidity while we continue to pursue neonatal infection risk factors. However, neonatal infection has occurred in the setting of inadequate maternal acyclovir suppression therapy. Clearly, properly conducted, placebo-controlled, randomised trials stratified accord- 
ing to type-specific immune status and frequency rates are warranted before new recommendations are made.

Finally, it is essential to recognise that current medical practice does not directly address the group at highest risk for neonatal transmission: HSV-susceptible women in the third trimester who are in HSV discordant relationships. ${ }^{9}$ Only routine serologic screening of both partners can begin to address prevention in this highest-risk patient.

\section{REFERENCES}

1. Whitley RJ, Nahmias AJ, Soong SJ, Galasso GG, Fleming CL, Alford CA. Vidarabine therapy of neonatal herpes simplex virus infection. Pediatrics 1980;66:495-501.

2. Whitley R, Arvin A, Prober C, et al. Predictors of morbidity and mortality in neonates with herpes simplex virus infections. The National Institute of Allergy and Infectious Diseases Collaborative Antiviral Study Group. N Engl J Med 1991;324:450-454.

3. Koskiniemi M, Happonen JM, Järvenpää AL, Pettay Ö, Vaheri A. Neonatal herpes simplex virus infection : a report of 43 patients. Pediatr Infect Dis J 1989;8:30-35.

4. Whitley RJ, Nahmias AJ, Visintine AM, Fleming CL, Alford CA. The natural history of herpes simplex virus infection of mother and newborn. Pediatrics 1980;66: 489-494.

5. Garland SM. Neonatal herpes simplex: Royal Women's Hospital 10-year experience with management guidelines for herpes in pregnancy. Aust NZ J Obstet Gynaecol 1992;32:331-334.

6. American Academy of Pediatrics Committee on Fetus and Newborn and Committee on Infectious Disease. Perinatal herpes simplex virus infections. Pediatrics 1980;66:147-149.

7. Arvin AM, Hensleigh PA, Prober CG, et al. Failure of antepartum maternal cultures to predict the infant's risk of exposure to herpes simplex at delivery. $\mathrm{N} \mathrm{Engl} \mathrm{J} \mathrm{Med}$ 1986;315:796-800.

8. American College of Obstetricians and Gynecologists. Perinatal herpes simplex virus infections. ACOG Technical Bulletin 122. Washington DC: American College of Obstetricians and Gynecologists, 1988.

9. Brown ZA, Selke S, Zeh J, et al. The acquisition of herpes simplex virus during pregnancy. N Engl J Med 1997;337:509-515.

10. Randolph AG, Washington AE, Prober CG. Caesarean delivery for women presenting with genital herpes lesions. Efficacy, risks, and costs. JAMA 1993;270:77-82.

11. Gibbs RS, Amstey MS, Lezotte DC. Role of cesarean delivery in preventing neonatal herpes virus infection. JAMA 1993;270:94-95.
12. Haddad J, Langer B, Astruc D, Messer J, Lokiec E. Oral acyclovir and recurrent genital herpes during late pregnancy. Obstet Gynecol 1993;82:102-104.

13. Frenkal LM, Brown ZA, Bryson YJ, et al. Pharmacokinetics of acyclovir in the term human pregnancy and neonate. Am J Obstet Gynecol 1991;164:569-576.

14. Merigan TC, Gregory DF, Petralli JF. Physical properties of human interferon prepared in vitro and in vivo. Virology 1966;29:515-522.

15. Sacks SL, The CZ, Lemchuck-Favel L. Serotyping of herpes simplex virus isolates: a comparison of BVDU sensitivities, indirect immunofluorescence with monoclonal antibodies, and indirect immunofluorescent with crossabsorbed rabbit antibodies. J Virol Methods 1983; 7:199-206.

16. Garland SM, Lee TN, Ashley RL, Corey L, Sacks SL. Automated microneutralization: method and comparison with Western blot for type-specific detection of herpes simplex antibodies in two pregnant populations. J Virol Methods 1995;55:285-294.

17. Vontver LA, Hickok DE, Brown A, Reid L, Gorey L. Recurrent genital herpes simplex virus infection in pregnancy: infant outcome and frequency of asymptomatic recurrences. Am J Obstet Gynecol 1982;143:75-81.

18. Brown ZA, Benedetti J, Ashley R, et al. Neonatal herpes simplex virus infection in relation to symptomatic maternal infection at the time of labour. N Engl J Med 1991;18:1247-1252.

19. Catalano PM, Merritt AO, Mead PD. Incidence of genital herpes simplex virus at the time of delivery in women with known risk factors. Am J Obstet Gynecol 1991;164:1303-1306.

20. Chuang T. Neonatal herpes: incidence, prevention and consequences. Am J Prev Med 1988;4:47-53.

21. Brown ZA, Vontver LA, Benedetti J, et al. Effects on infants of a first episode of genital herpes during pregnancy. New Engl J Med 1987;317:1246-1251.

22. van Everdingen JJE, Peeters MF, ten Have P. Neonatal herpes policy in the Netherlands. Five years after a consensus conference. J Perinat Med 1993:21:371-375.

23. Harger JH, Pazin GJ, Breinig MC. Current understanding of the natural history of genital herpes simplex infections. J Reprod Med 1986;31:S365-S373.

24. Infectious Diseases and Immunization Committee, Canadian Paediatric Society. Toward the rational management of herpes infection in pregnant women and their newborn infants. Can Med Assoc J 1992;146:1557-1560.

25. Wald A, Corey L, Cone R, Hobson A, Davis G, Zeh J. Frequent genital herpes simplex virus 2 shedding in immunocompetent women. Effect of acyclovir treatment. J Clin Invest 1997;99:1092-1097. 


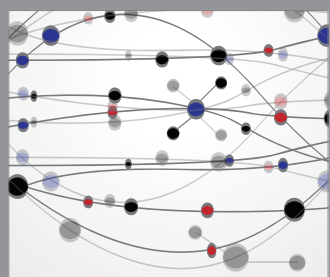

The Scientific World Journal
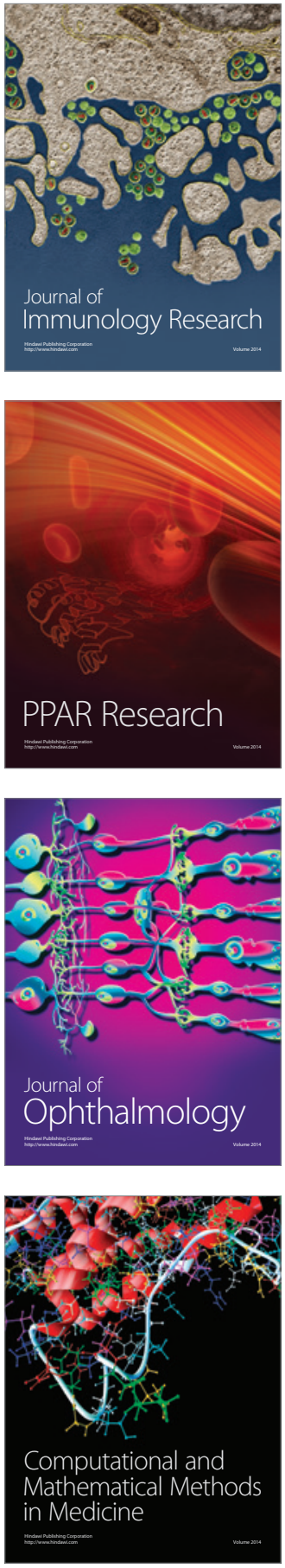

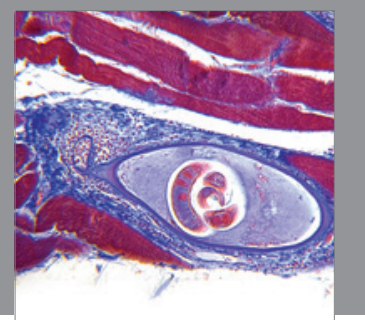

Gastroenterology

Research and Practice
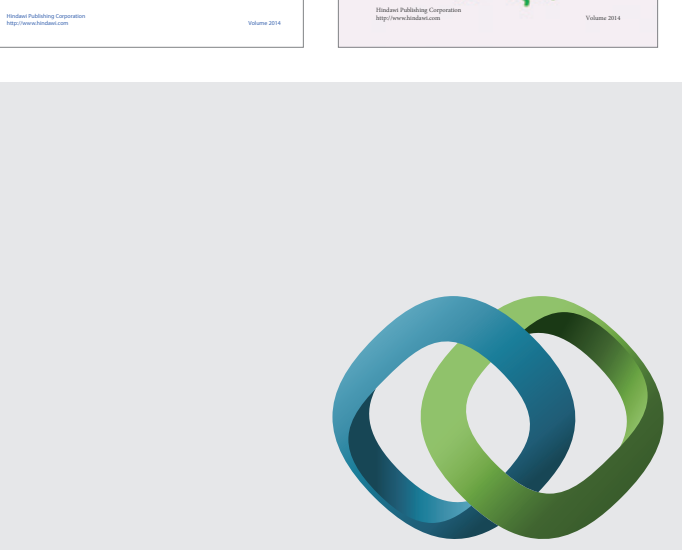

\section{Hindawi}

Submit your manuscripts at

http://www.hindawi.com
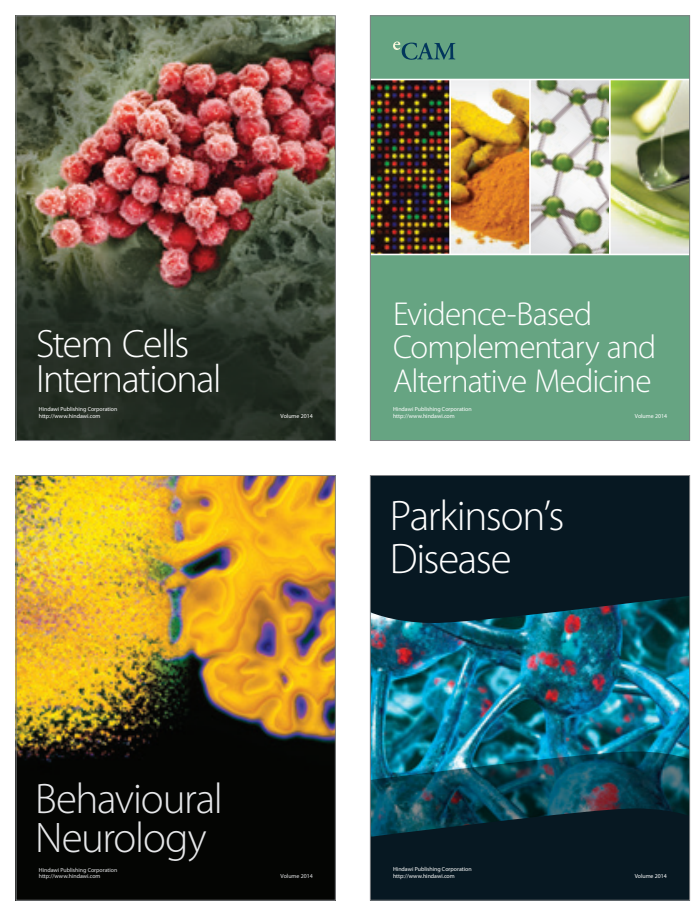

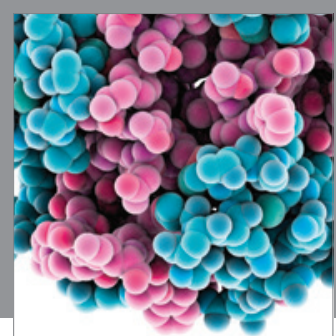

Journal of
Diabetes Research

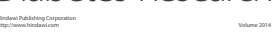

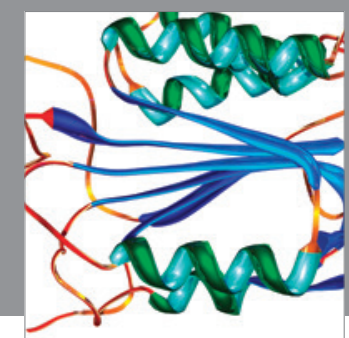

Disease Markers
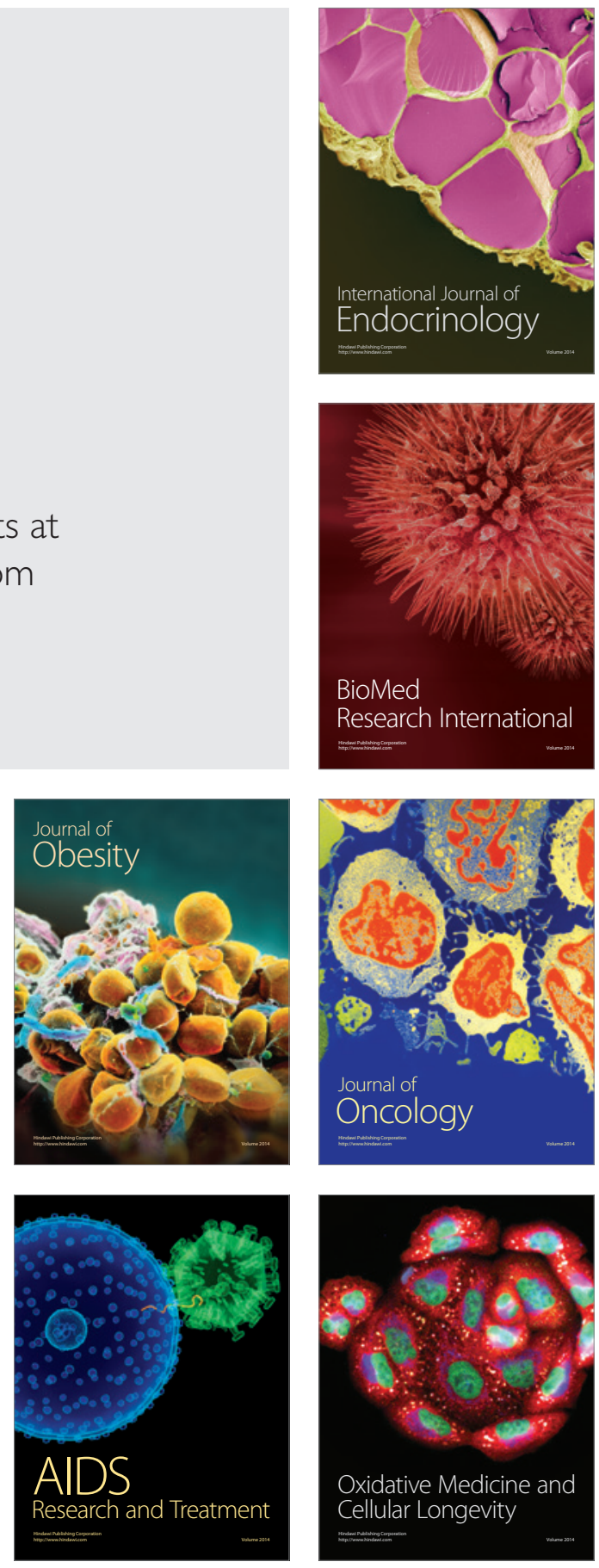